\title{
Errata: Fuzzy logic and optical correlation-based face recognition method for patient monitoring application in home video surveillance
}

\author{
Marwa Elbouz \\ Ayman Alfalou \\ ISEN Brest (Institut Supérieur, de l'Electronique et \\ du Numérique) \\ Département Optoélectronique \\ L@bISEN \\ 20 rue Cuirassé Bretagne, CS 42807 \\ Brest Cedex 2, Finistere 29228, France \\ E-mail: ayman.al-falou@isen.fr

\section{Christian Brosseau} \\ Université Européenne de Bretagne \\ Université de Brest \\ Lab-STICC \\ CS 93837, 6 avenue Le Gorgeu \\ Brest Cedex 3, 29238 France \\ [DOI: 10.1117/1.3602165]
}

This article [Opt. Eng. 50, 067003 (2011)] was originally published on 1 June 2011 with an error in the second author's name. The correct name is "Ayman Alfalou," as it appears above.

The manuscript was corrected online on 3 June 2011. The article appears correctly in print. 